\title{
Reduce, Reuse, Recycle: Visual Re- Representation of Design Engineering Knowledge
}

\author{
Emily CAREY ${ }^{\mathrm{a}, 1}$, Steve CULLEY ${ }^{\mathrm{a}}$ and Bruce ALLEN ${ }^{\mathrm{b} \dagger}$ \\ ${ }^{a}$ University of Bath, Claverton Down, Bath BA2 7AY, UK \\ ${ }^{b}$ Airbus, Pegasus House, Aerospace Avenue, Bristol, UK
}

\begin{abstract}
The exponential growth of data and information artefacts in engineering organisations are one of the biggest limitations for engineers reusing design knowledge. Approaches to support efficient information access are critical for design. In this paper, we demonstrate an exemplar "Super Document" principle using knowledge "Reduce, Reuse, Recycle" process. Evidence suggests such recycled representations can reduce the time engineers spend on gathering information from documents by up to $70 \%$. We outline the "Reduce, Reuse, Recycle" approach using empirical studies with in-service aerospace design engineers. We suggest visual representations better record and communicate information for design engineers, resulting in a time saving of $70 \%$ per design in document information seeking. The infographic "Super Document" created uses visual re-representation of large knowledge artefacts - reducing a 100-page design document to a single page. An insight into the next steps for this research concludes this paper.
\end{abstract}

Keywords. Design Engineering, Knowledge Management, Knowledge Reuse, Visual Information Representation, Aerospace In-Service.

\section{Introduction}

Studies investigating "case based" design engineering (DE) and the reuse of DE information found that solutions reuse between $75-90 \%$ of prior design [1][2] and up to $60 \%$ of DE time is spent on information resources [3]. To have successful reuse of prior design requires timely and appropriate information and knowledge provision [4][5]. Time is a critical element in the DE process for high value complex products such as aerospace in-service engineering, where minimising aircraft ground time is a customer priority [6][7]. However, many companies are still struggling with effective codification and storage of knowledge for reuse to reduce such design times [4][8]. In parallel to this DE attempts to maintain competitive edge have seen noticeable increases in product related service provision in industries such as aerospace [6][7][9]. This provision of service for products, amasses growing amounts of explicit data and tacit DE knowledge [7]. High value products with long lifecycles increase the longevity of data, feeding forward into new design and exacerbates the codification and storage problems. This poses unique repository accessibility and quality problems for reusing explicit

\footnotetext{
${ }^{1}$ Corresponding Author, Mail: [emcc20@bath.ac.uk].

$\dagger$ Author is Deceased, $2^{\text {nd }}$ April 2021.
} 
information and tacit knowledge. DE companies that provide in-service support find their teams having to utilise huge repositories of unstructured documents for information. This is cumbersome and time consuming, resulting in lower productivity and slower response to customers [7]. Approaches to support effective reuse of DE knowledge continue to be lacking in current technology provision [4]. The studies outlined in this paper illustrate an exemplary approach developed in partnership with an aerospace DE inservice team that reduced the amount of time engineers spent on document repository information by up to $70 \%$.

\section{Background}

Reuse for DE is significant, with an estimation of $75-90 \%$ being reuse from prior work [1]. More recent studies hold this still true, with prior information resources remaining one of the most important commodities for DE [2][3][6][8]. However deeper studies into the reuse of knowledge maintain the purpose of the reuse is critical for its effective capture and to make it useful in practice [5] but studies conclude there is no consensus how best to provide knowledge support for DE [4]. Studies state that to capture and reuse knowledge it first needs to be decomposed to simplify its complexity [8] or content codified appropriately [10]. In practice multipurpose information repositories do not satisfy the need for quality and timely information [5][10], an example is the common DE practice using large documents to meet regulatory approval. Without considerable effort this does not support the ease of access required for DE to handle use, reuse, and share knowledge [4][6][9].

Researchers accept there is human propensity for visual information assimilation [11] and development of visual support is prolific improving data analysis and learnings. Computational techniques applied to automate analysis are beneficial, but it remains that humans are far better at spotting atypical patterns and deducing meaning from visual representations [11]. The value of visual representations in supporting DE to better understand and communicate complex ideas has long been recognized [11] hence support should seek to capitalize on human visual capability in information support [2]. Recent information seeking surveys for DE evidence up to $80 \%$ of important information is visual [2]. This is particularly significant for the large unstructured types of information being amassed in in-service aerospace, where DE believe they are using information search strategies but are employing browsing techniques to glean knowledge [7].

A heterogenous mass of information resources and information types, including documents, reside within in-service DE teams [6][7][9]. This is critical to support repair designs in highly time pressured environments with stringent customer turn-around times to maintain aircraft and reduce the cost of aircraft ground time. Two example information audits reported in [6][7], found over 200 information resources being accessed regularly by the team. This highlights the significance of explicit resources such as unstructured document collections. A second audit of the growing document repository (140GB) details unstructured heterogenous media types with a visual content prevalence and textbased correspondence, highlighting the need for improved access.

Data is still increasing, for example aerospace products with service support are predicted to increase significantly (19,890 in 2011 to 39,780 by 2031) with little increase in manpower [6][7]. Alongside this repair documents alone are exponentially increasing 
as are the number of aircraft in-service per DE [7]. Research also suggests that as products evolve there is a reliance upon this technical information and prior captured DE solutions [2][9]. This makes the task of deciding upon information relevance increasingly complex and multifaceted. With the expected addition of 20,000 more aircraft by 2031 and extra 2,000 aircraft per engineer, our industry partners aim was to improve document information access to reduce the time it takes engineers to find relevant information [6][7].

\section{Experimental Studies and Results}

The studies outlined in this paper are part of a sequence of studies conducted with an inservice aerospace DE team. The overall research focused upon the importance of visual information to support engineering knowledge management (KM). A full report of the associated studies, experimental and analysis methods is reported in [6]. An overview is provided here due to space limitations and the extent of the studies reported.

\subsection{Significantly Informing Document Content}

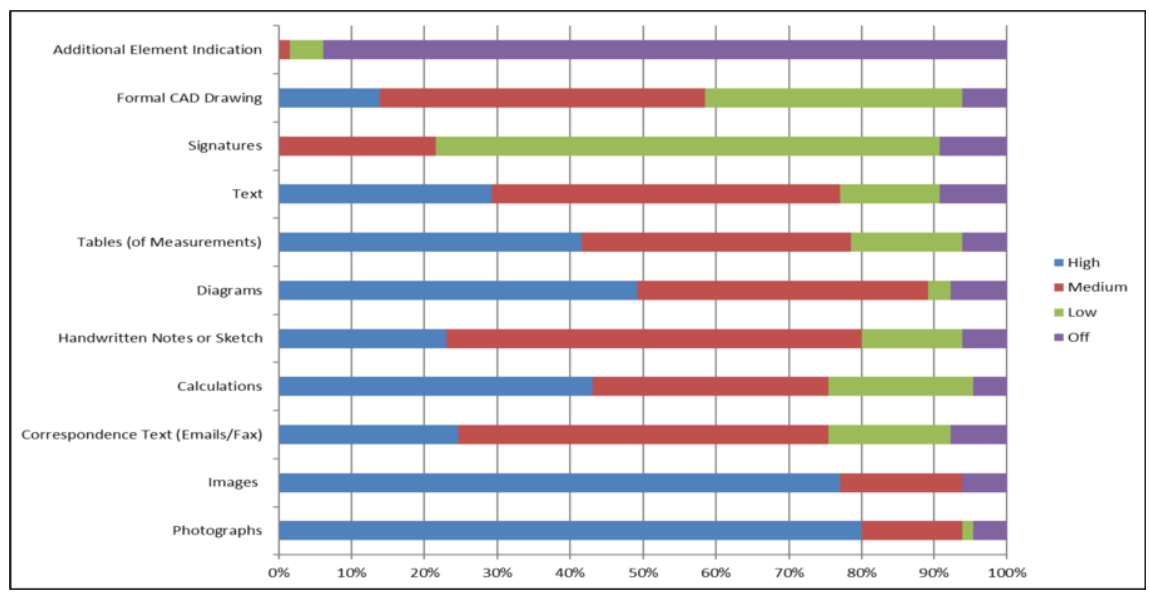

Figure 1. Ranked Importance of Document Elements.

Using prior reported document content audit results [6][7], a three part, international and multi-site survey (US, Germany, France \& UK teams) of in-service engineers was conducted with a $70 \%$ response rate. Insight was sought to deconstruct the "how", "why" and "what" within the document media was being used to create repair designs. The survey shows that DE perceive they are information seekers, but results demonstrate in documents "information browsing" techniques are predominantly employed.

The survey highlights that document repositories are a significant information asset reused in the aerospace in-service team (64\%). It indicates engineers typically process 20 repairs per week. For each repair 25-70 minutes is spent browsing document content with additional subsequent document references. This totals no less than 2-4 hours and 40 minutes each day spent in documents. This amounts to 10-23 hours per week spent on historic documents for each DE. Results show that for DE, visual media types are being accessed most frequently and are deemed to be most informing. Figure 1 below illustrates the "high" significance of "photographs", "images", "diagrams" and 
"calculations". Astonishingly, for in-service design the comparative value attributed to "formal CAD Drawings" is low relative to other media forms.

\subsection{Example Document Knowledge Story}

This raised further question as to the purpose of the information reuse [5] and how best to reduce information complexity to simplify and improve its usability [8][10], both critical to KM solutions and their successful uptake [6]. A workshop investigated DE thinking in deciding upon the relevance of documents. A scenario mocked up a repair design case and deconstructed document media to be sequenced on storyboards eliciting information decision stories. An example deconstructed decision story is shown in Figure 2 below, illustrating key media and thinking sequences. The tacit value of both the textual and visual elements in this single information story is evident, illustrating the underpinning information decision-making process. Decision theory was applied in analysis as described in [6], but the stories highlight the human information transactions being undertaken and informative value of media types for recycling DE documents efficiently.

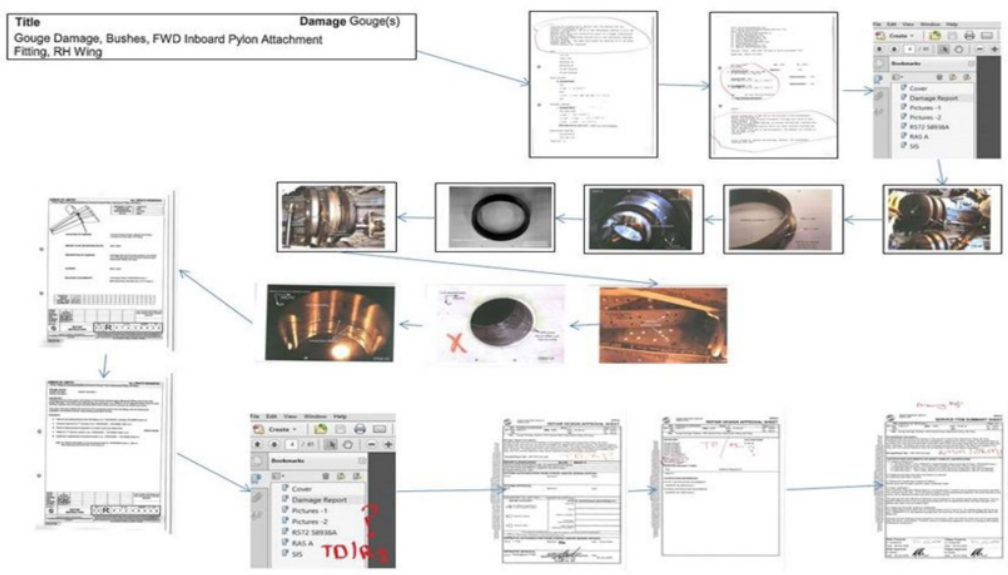

Figure 2. Example Informing Story.

\subsection{Design Engineering Information Relevance Evaluation}

Evaluations examined the usefulness of reduced document media combinations and alternative document representations (static and interactive solutions) with the entire UK in-service team in individual interviews. In interview, five differing document media combinations were provided with limited viewing time, followed by short survey questions. This assessed the information assimilated for each combination in 4 objective and 4 subjective design related questions. Results indicate that much reduced information media provides similar informative value for case-based reasoning but results in faster design decision making and proposes useful information utilization patterns for coding design knowledge. Significantly, reduced documents produced confident information relevance decisions $100 \%$ of the time if visual media was represented, but without visual media this dropped to $45 \%$, highlighting the importance of visual representations for DE decisions. However, removing text created an inability to make decisions, suggesting supporting DE to "information satisfice" is critical. 


\subsection{Visual "Super Document" Re-Representation}

Secondary evaluations recycle the information utilisation patterns in Carey [6] to create exemplar intervention as shown in Figure 3. This visual representation is an example of how over 100 pages of DE document can be efficiently summarised in visual 1-page "Super Document" infographics [12]. This can reduce the time DE spend on document information, coding minimal text for understanding with rich visual media to enable confident decisions upon its relevance and reducing the time required to recycle past design cases by up to $70 \%$. Experiments indicate the value of the visual elements in this representation, but do highlight that much of the textual information is already aquired by DE prior to viewing [6][12], suggesting even more efficient information trails exist.

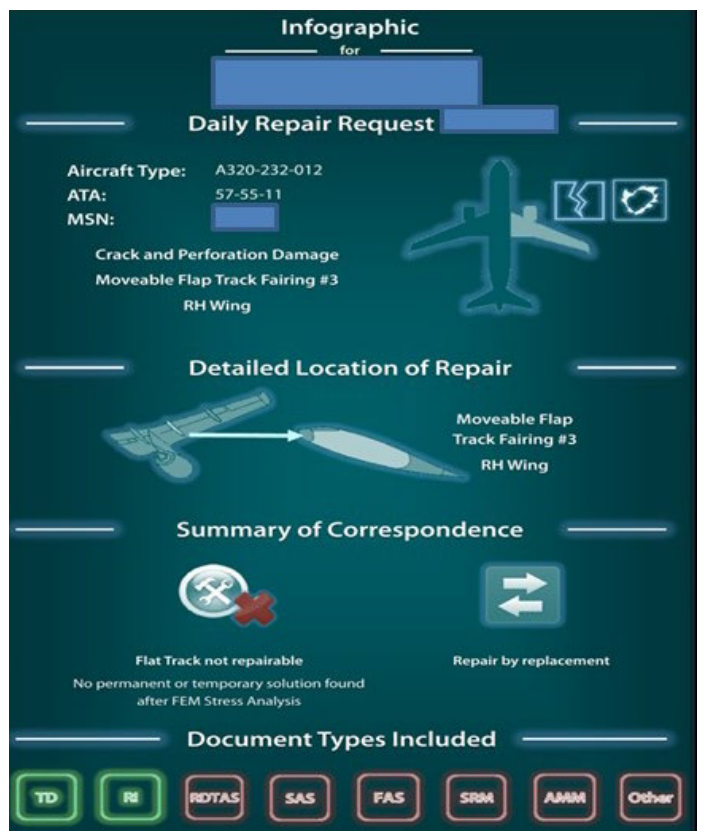

Figure 3. Recycled "Super Document” Infographic.

\section{Discussion and Conclusions}

DE documents are large heterogeneous data that cost DE significant time to understand their content [6][7][9][10]. However due to the time pressured environments they work in and the sheer scale of information amassed, engineers in this study have been shown to "information satisfice" and to enable reasonable decisions about information relevance to their current task [6]. If DE are going to be able to continue to work efficiently in the current and future manufacturing climate of industry 4.0, they need to be supported to better recycle the resources they have [2][4][5][6][7]. This means both efficiently capitalizing upon the rich explicit knowledge resources they amass during the lifecycle of a product and to optimizing the quality of the current DE outcomes [6][8][9]. This will require development of support to overcome the human and technological challenges this task poses. One such method demonstrated in this paper is to produce a "Super Document" 1-page summary from over 100 pages of design engineering documents [6]. Reducing 
the amount of information that a DE needs to spend time on to be able to reuse and recycle their knowledge. The 'super document' capitalizes upon the value visual representations hold in presenting complex DE information [2][6][11] and minimal target textual cues, cutting the time consuming "reading in depth" processing.

Future work needs to understand best how to "recycle" information for DE teams, using visual representations that are static such as infographic and interactive visualization solutions. For maximum efficiency and quality this should also investigate the coding of wider information utilization patterns that are recycled in DE processes and address legacy data challenges. This is important as additional efficiencies could also be in the quality and consistency of DE repairs produced from information. Reducing to succinct or minimal information resources provides value for international teams, where sharing sensitive customer information with reduced bandwidth over networks can create access and security problems but also overcome barriers in multilingual international DE teams.

\section{Memorandum and Acknowledgements}

The work reported in this paper is dedicated to the deceased Bruce Allen who contributed much of his time and energy, to support facilitating its creation. It has been undertaken with support from the Engineering and Physical Sciences Research Council's (EPSRC) and the Innovative Manufacturing Research Centre at The University of Bath (grant reference $\mathrm{EP} / \mathrm{E} 00184 \mathrm{X} / 1)$.

\section{References}

[1] Vijaykumar G, Chakrabarti A, Understanding the Knowledge Needs of Designers During Design Process in Industry. Journal of computing and information science in engineering. 2008. Vol;8:1.

[2] Qin H, Wang H, Johnson A, Understanding the information needs and information-seeking behaviours of new-generation engineering designers for effective knowledge management. Journal of Information Management. 2020. Vol;72(6):853-868.

[3] Robinson MA, An empirical analysis of engineers' information behaviors. Journal of the American Society for Information Science and Technology. 2010. Vol;61(4):640-658.

[4] Wang Y, Yu S, Xu T, A user requirement driven framework for collaborative design knowledge management. Advanced Engineering Informatics. Vol;33:16-28.

[5] Markus LM, Towards a Theory of Knowledge Reuse: Types of Knowledge Reuse Situations \& Factors in Reuse Success. Journal of Management Information Systems. 2001. Vol;18(1):57-93.

[6] Carey E, Visually Informed Support for Design Engineering Decisions [PhD Thesis]. [Bath] University of Bath; 2016.

[7] Carey E, Culley S, Weber F, Establishing Key Elements for Handling In-Service Information and Knowledge. In: Proceedings of the $19^{\text {th }}$ International Conference on Engineering Design; 2013 Aug 1922 Seoul, Korea: Design Society; 2013. Vol;6:11-20.

[8] Peng G, Wang H, Zhang H, Zhao Y, Johnson A, A collaborative system for capturing and reusing incontext design knowledge with an integrated representation model. Advanced Engineering Informatics 2017. Vol;33:314-329.

[9] Jagtap S, Johnson A, In-Service Information Required in a Redesign Task: An Analysis of Documents from the Aerospace Industry. Research in Engineering Design. 2011. Vol;22(4):207-221.

[10] Barton J, Love D, Retrieving designs from a sketch using an automated GT coding and classification system. Journal for Production Planning \& Control. 2005. Vol;16(8):763-773.

[11] Hendersen K, Flexible Sketches and Inflexible Data Bases: Visual Communication, Conscription Devices, and Boundary Objects in Design Engineering. Science. Technology, \& Human Values. 1991. Vol;16(4)448-473.

[12] Lockett A, Infographics for Engineering Design [Masters Dissertation]. [Bath]; University of Bath:2016. 